\begin{tabular}{|l|l|l||}
\hline \multicolumn{2}{|c|}{ PublisherInfo } \\
\hline \hline PublisherName & $:$ & BioMed Central \\
\hline \hline PublisherLocation & $:$ & London \\
\hline \hline PublisherImprintName & $:$ & BioMed Central \\
\hline \hline
\end{tabular}

\title{
Adenoviral topoisomerase II $\alpha$ gene transfer increases sensitivity of resistant cancer cells
}

\begin{tabular}{|l|l|l||}
\hline \multicolumn{2}{|c||}{ ArticleInfo } \\
\hline \hline ArticleID & $:$ & 3640 \\
\hline \hline ArticleDOI & $:$ & $10.1186 /$ bcr-1999-66618 \\
\hline \hline ArticleCitationID & $:$ & 66618 \\
\hline \hline ArticleSequenceNumber & $:$ & 60 \\
\hline \hline ArticleCategory & $:$ & Paper Report \\
\hline \hline ArticleFirstPage & $:$ & 1 \\
\hline \hline ArticleLastPage & $:$ & 4 \\
\hline \hline & $:$ & RegistrationDate : 1999-11-1 \\
\hline ArticleHistory & $:$ & OnlineDate \\
\hline \hline ArticleCopyright & $:$ & Current Science Ltd1999-11-1 \\
\hline \hline ArticleGrants & $:$ & \\
\hline \hline ArticleContext & $:$ & 1305811 \\
\hline \hline
\end{tabular}




\title{
Keywords
}

\author{
Adenovirus, breast cancer cells, etoposide, topoisomerase II
}

\section{Introduction}

Cellular resistance to chemotherapeutic agents is a major problem in cancer treatment. There are a number of possible mechanisms of drug resistance, one of which is changes in the level of topoisomerase II (topo II $\alpha$ ) gene expression. The topo II $\alpha$ nuclear enzyme is crucial for cell survival, being involved in many essential cellular processes such as DNA replication. The enzyme is also a target for a number of key chemotherapy drugs including adriamycin and etoposide. These drugs stabilise the enzyme-DNA cleavable complex which in turn initiates cellular death processes. Therefore, alterations in the topo II $\alpha$ enzyme can lead to drug resistance. One such alteration is a decrease in the level of enzyme, which leads to reduced amounts of topo II-DNA complex, thus less drug binding and less cell death. Conversely, the cytotoxicity of topo II targeting drugs increases as the level of topo II $\alpha$ enzyme in the cell increases. Etoposide-resistant MDA-VP human breast cancer cells express lower amounts of enzymatically active and drug-sensitive topo II than do MDA parent cells, suggesting that the low level of topo II $\alpha$ is the mechanism of resistance.

\section{Aims}

To determine whether transfer of a normal topo II $\alpha$ gene into MDA-VP cells can increase topo II gene expression, topo II $\alpha$ protein production, and cell sensitivity to etoposide.

\section{Comments}

This is a well performed study investigating a novel method of circumventing one method of drug resistance. It has been shown that low levels of topo II $\alpha$ protein correlate with tumour response to the topo II inhibitors, and so this technique of infecting the cells with the topo II $\alpha$ gene opens up the possibility of increasing the efficacy of the topo II inhibitors against a range of tumour types. Its seeming preference for cells with low levels of the protein indicates the possibility of a degree of cell selectivity, thus limiting normal cell toxicity. Unfortunately, tumour cells have often developed, and can 
continue to evolve, secondary mechanisms to escape the toxic effects of chemotherapy drugs, and so increasing the levels of topo II $\alpha$ protein may in itself not be sufficient to achieve tumour eradication. The next step will be to move from cell lines to the tumour xenograft model and it will be fascinating to follow the authors progress.

\section{Methods}

Human embryonic kidney cells $(n=293)$ transformed with adenovirus type 5 were cotransfected by the shuttle vector pAvCvSv-hTopII (containing the key topo II gene fragments) and the pBHG10 packaging vector. Infectious recombinant adenovirus plaques were isolated and purified. Thus, a recombinant adenovirus, Ad-hTopo II $\alpha$, containing the human topo II $\alpha$ gene, had been constructed. MDA-VP breast cancer cells (etoposide-resistant) were then infected by various concentrations of adenovirus and various doses of etoposide were added. Anti-proliferative activity was then measured.

\section{Results}

The presence of the topo II $\alpha$ gene and appropriate adenoviral sequences in the recombinant Ad-hTopo II $\alpha$ was confirmed by PCR and restriction enzyme digestion. The parent MDA cells and the resistant MDA-VP cells were characterised. The major difference between the two was the level of topo II $\alpha$ expression (decreased by $>85 \%$ in the resistant cells) which in turn lead to a decrease of around $80 \%$ in topo II-DNA complex formation in the presence of etoposide. It was also shown that the topo II $\alpha$ that was present in the resistant cells was functionally active and thus was not a non-reactive mutant form. After infection with Ad-hTopo II $\alpha$, topo II mRNA expression in MDA-VP cells increased in a dosedependent manner by 7.4-fold. Topo II protein production was increased 5.9-fold, and cellular sensitivity to etoposide was increased 4.5 times compared with control transfected cells, an increase that was also dose-dependent. Infection of normal human embryonic lung cells and human fibroblast cells with Ad-hTopo II $\alpha$ did not enhance the expression of topo II or increase their sensitivity to etoposide.

\section{Discussion}

These data suggest that the adenoviral vector mediated transfer of the topo II $\alpha$ gene can increase etoposide sensitivity in etoposide-resistant breast cancer cells. In addition, there appeared to be selective topo II $\alpha$ up-regulation in tumour cells expressing low levels of topo II $\alpha$. This opens up the possibility of an improved therapeutic index of etoposide, as normal cells would not suffer from increased sensitivity to etoposide. Why normal cells are not affected by infection with the adenovirus is unclear, but it might be related to tight cellular control of sustained increases in topo II $\alpha$ protein levels in normal cells. 


\section{References}

1. Zhou Z, Zwelling LA, Kawakami Y, An T, Kobayashi K, Herzog C, Kleinerman ES: Adenovirusmediated human topoisomerase II $\alpha$ gene transfer increases the sensitivity of etoposide-resistant human breast cancer cells. Cancer Res. 1999, 59: 4618-4624. 